

\title{
Agroecología: Una alternativa sostenible para la pequeña agricultura en un escenario post COVID19
}

\section{Agroecology: A sustainable alternative for small agriculture in a post-COVID19 scenario}

\author{
Josue Otoniel Dilas-Jiménez \\ Universidad Nacional Autónoma de Tayacaja Daniel Hernández Morillo. Perú. \\ Dilma Ascurra -Toro ORCID \\ Yelison Business S.A.C. Jaén. Perú.
}

\section{RESUMEN}

La actual tendencia del mundo con el incremento poblacional, la contaminación ambiental, el cambio climático, los desafíos por reducir la pobreza y el hambre de las personas, así como un nuevo escenario originado por el COVID19, hace que en los países se deba tener especial atención a estrategias o enfoques que desde distintos ángulos contribuyan a generar soluciones sostenibles. Ante ello, la agroecología o agricultura ecológica se presenta como una alternativa factible para la pequeña agricultura tanto en lo ambiental, lo social y lo económico; conjugándose con actividades complementarias y sumamente importantes como las bioferias o mercados agroecológicos que traen beneficios win to win tanto para el pequeño agricultor como para el consumidor en las ciudades, sin embargo, estos deberán adaptarse a un nuevo modelo de comercialización en entornos virtuales y comercio electrónico..

Palabras Claves: COVID, pobreza, agricultura ecológica, bioferias. 


\begin{abstract}
The current trend in the world with the increase population, pollution, the climate change, the challenges of reducing poverty and hunger of people, as well as a new scenario caused by COVID19, means that countries should have special attention to strategies or approaches that from different angles contribute to generate sustainable solutions. Given this, the agroecology or ecological agriculture is a feasible alternative for small agriculture both in environmental, social and economic terms; conjugating with complementary and important activities such as ecological fairs or agroecological markets that bring win-to-win benefits for both the small farmer and the consumers, however, these must be adapted to a new marketing model in virtual environments and e-commerce.
\end{abstract}

Keywords: COVID, poverty, ecological agriculture, ecological fairs.

\section{INTRODUCCIÓN}

Con el incremento de la población mundial de 1,6 billones en 1900, 6.1 billones en el 2000, a la actualidad con 7.8 billones y una proyección de 9 billones para el 2050 (Akaev \& Sadovnichii, 2010; PopulationCity, n.d.), nacen varios desafíos en el mundo, entre ellos aquellas formas de producción limpia como la Agroecología como un enfoque de producción en la agricultura, desafíos que buscan dar seguridad alimentaria al mundo y a la vez no perjudicar el medio ambiente y la salud de las personas y todo ser vivo albergado en nuestro planeta. Si bien la revolución verde creciente en los 60s fue una solución plausible a la necesidad de alimento para el mundo, esta estuvo enmarcada en un enfoque de productividad, esto es la agricultura intensiva o agricultura convencional, sin embargo, la agricultura en este enfoque ha contribuido de manera importante a la generación de Gases de Efecto Invernadero en el ambiente lo cual a su vez produce el conocido cambio climático (González Elizondo et al., 2003) que tiene sus efectos negativos en la salud de los seres vivos en el planeta (Pabón \& Nicholls, 2005) siendo entonces importante la implementación de prácticas ecológicas en la agricultura (García et al., 2006). Otros enfoques sostenibles como 
los sistemas agroforestales que buscan diversificar los ingresos de los agricultores, así como recuperar y dar sostenibilidad a los suelos y el ambiente mediante la captura y almacenamiento de carbono, también pueden ser congruentes con la producción agroecológica (Alegre, 2017; Dilas-Jiménez \& MugruzaVassallo, 2020; Rojo-Martínez et al., 2003), no necesariamente bajo una producción orgánica certificada (Barham \& Weber, 2012).

Ante ello, posterior al auge de la tecnologías propagadas por la revolución verde, avizorando las consecuencias de esta, en la década de 1980 la agroecología surgió como prácticas agrícolas, originando movimientos agroecológicos en las década de 1990, adicionalmente con lo cual nacen también los movimientos de cultivos orgánicos, que es otro concepto de agricultura cuyos conceptos pueden ser homologados en algunos casos(Callicott, 1988; Cuesta et al., 1998).

El Perú no ha sido ajeno a la corriente agroecológica mundial, aún más cuando nuestro modelo productivo está basado en pequeña agricultura y agricultura de subsistencia. En la década de los 80s a fines se constituye la Red de Agricultura Ecológica (RAE), con la cual se puede decir que inicia el movimiento agroecológico peruano (Felipe-Morales, 2020; Fernando Alvarado; Siura Saray, 2015), sin embargo los pequeños agricultores agroecológicos que ofertaban sus productos en las bioferias se han visto afectados por el actual escenario originado por el COVID19.

Para la realización del presente estudio, se realizó una búsqueda de información académica tanto histórica como actual sobre la agroecología, para ello se utilizó bases bibliográficas como ScienceDirect, Springer, SciElo, entre otras. El objetivo fue Conocer los avances históricas y actuales de la agroecología y su aplicación en la pequeña agricultura peruana.

\section{Desarrollo}

A fin de generar conclusiones y aportes aplicables a la temática de la revisión, se optó por incluir información referente sobre algunos casos vinculados a la aplicación práctica de la agroecología y su enfoque en la pequeña agricultura peruana.

\section{La agroecología como movimiento en el mundo}

Se reporta que el término agroecología se usó en el mundo ya en la década de 1930, sin embargo hasta 1960 este término se 
refería a una disciplina puramente científica, desarrollándose diferentes ramas de la agroecología, considerándose 3 enfoques principales: (i) investigaciones a escala de parcela y campo, (ii) investigaciones a escala de agroecosistema y finca, y (iii) investigaciones que abarcan todo el sistema alimentario (Wezel et al., 2009).

La Real Academia Española relaciona a la agroecología con el concepto de agricultura ecológica o también agricultura alternativa como lo refieren otros autores, así, según Altieri (1987) se define como cualquier enfoque de la agricultura que busque dar rendimientos sostenidos haciendo uso de tecnologías de gestión ecológicamente racionales, donde sus estrategias se basan en conceptos ecológicos como: reciclaje de nutrientes y materia orgánica, flujos de energía cerrados, búsqueda del equilibrio de poblaciones y el uso múltiple del paisaje.

Se ha llegado a afirmar que la agroecología puede desempeñar un papel importante en la búsqueda de soluciones para los desafíos mundiales de la lucha contra el hambre y la contaminación mundial y cambio climático, asimismo, se ha podido afirmar que las prácticas agroecológicas aplicados por pequeños productores constituyen más del 50\% de la producción de alimentos del mundo y que se producen en el $20 \%$ de las tierras agrícolas, pudiendo incluso duplicar la producción de los alimentos en el mundo en un plazo de 10 años (Migliorini \& Wezel, 2017). Así, la agroecología ha movido el mundo académico a través de la investigación agrícola y la extensión agraria donde se ha involucrado ONGs, el estado y organizaciones privadas, siendo compatible para la pequeña agricultura o la agricultura de subsistencia (Epule \& Bryant, 2017) ya que se enfoca en lo siguiente (M. A. Altieri \& Yurjevic, 1992): (i) Es aplicable a las familias de pequeños agricultores con metodologías al tamaño de sus necesidades, (ii) usa técnicas de bajos insumos activando así un movimiento social de alta participación popular, (iii) sus técnicas no cuestionan la lógica de los campesinos y se combinan con el conocimiento tradicional, (iv) no pretende modificar el ecosistema del campesino pero buscando optimizar la producción, (v) la aplicación del enfoque es económicamente viable al buscar minimizar los costos de producción, y optimizar el uso de la unidad productiva. Sin embargo, en la década de 1990 se inició también otra corriente de producción agrícola homóloga a la agroecología, nos referimos a la 
“producción orgánica". En el 2008 la Asamblea General de IFOAM (IFOAM, 2008), que es el organismo internacional sobre el tema, define la agricultura orgánica como un sistema de producción que mantiene la salud de los suelos, los ecosistemas y las personas. Se basa en procesos ecológicos, biodiversidad y ciclos adaptados a las condiciones locales, más que en el uso de insumos con efectos adversos. Como vemos este concepto tiene mucha similitud con el concepto de agroecología, así también guardan similitud en sus prácticas y principios, pero del análisis de ambas aplicaciones en la agricultura se ha encontrado que éstas se diferencian básicamente en 2 aspectos: (i) la producción con certificación y (ii) el no uso de pesticidas y fertilizantes químicos, que son exigibles y obligatorios para usar un sello orgánico, pero, en otros aspectos como labranza del suelo, nutrición del suelo, elección de variedades, manejo de malezas, rotación de cultivos, manejo de plagas $y$ enfermedades y otros es compatible tanto para la agricultura orgánica como para la agroecología (Migliorini \& Wezel, 2017).

Una reciente publicación hacer referencia a que la actual crisis por la pandemia originada por el COVID19 ha demostrado la estrecha vinculación entre la salud humana, la salud animal y la salud ecológica, y que, la dependencia mundial a pocos cultivos commodities como el trigo, arroz y maíz que representan más del $50 \%$ de las calorías consumidas en el mundo, lo cual ha hecho que se popularice la forma de producción en monocultivos, sin embargo, estos resultan ser altamente vulnerables a las epidemias de plagas y enfermedades para los cultivos, además que, originan una fuerte dependencia de alimento a gran parte de la población de escasos recursos. Esto, en un ambiente de pandemia, por el cierre de fronteras y otras limitaciones como transporte y logística, incrementa la vulnerabilidad de las personas de más bajos recursos, así como a los países que depende de mano de obra de migrantes (Miguel A. Altieri \& Nicholls, 2020).

Finalmente, si bien la producción agroecológica no tiene una norma internacional o certificación exigible, los países suelen tener algunos reconocimientos la implementación de "sistemas de garantía participativos (SGP)" para este tipo de modelos productivos. Cabe precisa que otras certificaciones como Rainforest Alliance podrían ser mucho más compatibles con el enfoque de producción agroecológica (Barham \& Weber, 2012), más aún, 
cuando estudios han encontrado que la agricultura orgánica por la especialización que esta requiere conlleva a elevar los costos de producción, menor producción por unidad de área, así como los gastos en la obtención y mantenimiento de la certificación, lo cual puede tener impactos económicos no muy alentadores para la economía del pequeño productor (Beuchelt \& Zeller, 2011; Laura S., 2013; Poudel et al., 2015). Por ello, una agricultura sustentable debe basarse en lo que se denominaría principios ecológicos no en recetas, como (Felipe-Morales, n.d.): (i) Adaptar los cultivos al medio, (ii) diversificar los cultivos e incorporar las crianzas, (iii) concebir de manera integral la fertilidad del suelo, (v) favorecer el reciclaje de nutrientes del suelo y la materia orgánica, (vi) enfrentar las causas y no los síntomas en la protección vegetal, (vii) fomentar el control biológico, (viii) conservar el agua y el suelo, (ix) revalorar el conocimiento tradicional.

\section{Movimiento agroecológico en Perú}

Si bien en la década de los 80 s a través de trabajo de Centro IDEAS se instalaron parcelas demostrativas agroecológicas, el 1989 con la creación de la Red de Agricultura Ecológica (RAE) es que se podría decir que inicia el movimiento agroecológico en el Perú (Felipe-Morales, 2020). A partir de ello entre 1989 al 2013 se fundaron las bases institucionales del movimiento agroecológico en el Perú, asî en 1990 se crea la Red de Acción en Agricultura Alternativa (RAAA), en 1998 de conforma la Asociación Nacional de Productores Ecológicos del Perú (ANPEPERU), en 1999 se da lugar a la creación de la Bioferia en Miraflores, en el 2001 se crea la Comisión Nacional de Productores Orgánicos (CONAPO), en el 2004 se crea la Asociación de Consumidores Ecológicos, en el 2005 se crea el Consorcio Agroecológico del Perú, en el 2008 el estado peruano promulga la Ley $\mathrm{N}^{\circ}$ 29196, Ley de Promoción de la Producción Orgánica y Ecológica, la cual a su vez en el 2012 da lugar a la aprobación de su reglamento mediante Decreto Supremo $\mathrm{N}^{\circ}$ 010-2012-AG, ya reciente en el 2019 se promulga la Ley $N^{\circ} 30983$, Ley que modifica la Ley $\mathrm{N}^{\circ}$ 29196, ley de promoción de la producción orgánica o ecológica, a fin de desarrollar la certificación de productos orgánicos producidos por pequeños productores (Estado-Peruano, 2008， 2012， 2019; Felipe-Morales, 2020; Fernando Alvarado; Siura Saray, 2015). 
Desde 1999 con la creación de la Bioferia de Miraflores en Lima se inicia un proceso de concientización a la población peruana, principalmente en una ciudad que centraliza casi un tercio de la población nacional. Sin embargo, este proceso no es muy visible sinó hasta que la sociedad Peruana de Gastronomía (APEGA), asociación sin fines de lucro fundada por Gastón Acurio, lleva adelante la denominada Feria Gastronómica MISTURA que tuvo su primera versión en el año 2008, feria que se desarrolló regularmente hasta el año 2016. Además de la feria MISTURA que tuvo impacto nacional e internacional, también se promovió el feria dominical de productores regionales que se constituyó en una estrategia para que pequeños productores organizados de todo el Perú tuvieran un espacio para la difusión y venta directa de sus productos (Fernando Alvarado; Siura Saray, 2015).

A partir de estos avances históricos del movimiento agroecológico en el Perú y las implicancias en políticas públicas que ha tenido en el Perú, se añade a este estudio algunas experiencias o casuísticas vinculadas a la agroecología y las ferias agroecológicas o bioferias, a continuación:

\section{Experiencias agroecológicas por el Programa Sierra Productiva}

De lo obtenido y analizado en el portal web del Programa Sierra Productiva (Sierra-Productiva, n.d.), este programa ha sido implementado en Perú por organizaciones campesinas de base, con la facilitación del Instituto para una alternativa Agraria-IAA, que es una asociación civil sin fines de lucro. El programa surgió en Cusco en 1994, ésta se ha podido replicar en 17 regiones del país contribuyendo a la mejora de la calidad de vida en 60 mil familias, que han logrado enfrentar a la pobreza. El Programa hace que se produzca una combinación sinérgica entre conocimientos innovadores con la implementación de tecnologías para el aprovechamiento de sus recursos naturales (agua, tierra, cobertura vegetal), sus recursos productivos (semillas, cultivos, crianza) y recursos culturales. Un aspecto clave en el éxito del programa consiste en la capacitación de campesino a campesino conducida por YACHACHIQ, que son campesinos líderes que capacitan con el método "aprender haciendo". Es destacable las técnicas/tecnologías promovidas/desarrolladas por este programa, en 2 grandes temáticas, según lo siguiente: (i) Tecnologías productivas y de 
transformación: Agroforestería, bomba de Ariete, elaboración de abonos orgánicos, establo mejorado, forraje verde hidropónico, huerto fijo a campo abierto, módulo de crianza de gallinas, módulo de crianza de cuyes, miniparcelas de granos y tubérculos andinos, pastos asociados cultivados, riego por aspersión, riego por goteo, productos lácteos. (ii) Tecnologías para mejora de la vivienda familiar: Agua segura para consumo humano con nanofiltro, baño seco, biodigestor para producir biogás, cocina mejorada con horno y agua caliente, cosina solar, ecobaño, refrigeradora ecológica, refrigeradora ecológica, terma solar.

De las técnicas/tecnologías antes indicadas, todas ha sido aplicadas por familias de pequeños productores en zonas andinas y otras regiones en el Perú; varias de ellas corresponden a tecnologías básicas que contribuyen a la seguridad alimentaria y mejorar la calidad de vida de pequeños productores (Sierra-Productiva, 2016). Así también, técnicas básicas como el riego por goteo o por aspersión, han permitido hacer sostenible y rentable la ganadería, así como el suministro de hortalizas durante todo el año, cuando antes sólo era posible en época de lluvias (sólo 3 a 4 meses en un año). Por tanto, estas técnicas/tecnologías promovidas por el Programa Sierra Productiva son altamente compatibles con la producción agroecológica, pudiendo complementarse además con tecnologías que pueden tener un mayor nivel tecnológico originados a partir de la investigación aplicada y testeadas en el Perú, tal es el caso de la técnicas de biofertilización con micorrizas arbusculares o aplicación de Rhizobium por ejemplo (Davies et al., 2005; Nuñez et al., 2005; Santillana et al., 2016), así como el establecimiento de cultivo en sistemas agroforestales (Dilas-Jiménez \& Mugruza-Vassallo, 2020).

\section{Agroecología en la finca Bio-} Agricultura Casa Blanca

A partir de apuntes tomados en un curso de Agroecología Avanzada en la Universidad Nacional Agraria la Molina y video (Felipe-Morales, 2020; MINAM, 2015), Bio-Agricultura Casa Blanca, es una finca implementada con enfoque Agroecológico, de propiedad de 2 ingenieros agrónomos, Carmen FelipeMorales (especialista en suelos) y Ulises Moreno (especialista en fisiología de plantas), ubicado en la zona de Pachacámac (valle de Lurín), Lima, Perú. Esta finca ha sido implementada hace más de 30 años, en un área de 1ha, cuenta con 
un enfoque Agroecológico en todos sus extremos, así como de economía circular. Se ha consolidado como un lugar favorito de docentes y estudiantes de colegios y de universidades, investigadores y otro público que visitan sus instalaciones para conocer la experiencia que se imparte; es así que entre el 2001 hasta el 2015 tiene registrado la recepción de $217 \mathrm{mil}$ visitantes entre nacionales y extranjeros. Esta finca, no sólo destaca por su enfoque Agroecológico sino por llevar una filosofía de hacer agricultura de manera sostenible (social, económico y ambiental) donde sus dueños (Ingenieros agrónomos) pueden mostrar con hechos que "si es posible implementar este tipo de agricultura", constituyéndose así en una inspiración para jóvenes estudiantes y profesionales. A la actualidad, Bio-Agricultura Casa Blanca ha diversificado sus productos y servicios, ofreciendo entre otros: producción agrícola, venta de cuyes, venta de frutas y otros productos de la finca, visitas guiadas, participación en bioferias, cursos de capacitación, servicio de alimentación a pedido (pachamanca de cuy), espacios para tesis y otras investigaciones, elaboración y venta de helados y otros productos de consumo elaborados.
Entre los aspectos o tecnologías que puedo destacar en esta experiencia, puntualmente son: (i) La implementación de la finca en sistema agroforestal: que da una función ecológica a la finca, aporta sombra $y$ generación de un microclima adecuado, así como de un aporte paisajístico y beneficios ambientales (Dilas-Jiménez \& Mugruza-Vassallo, 2020); (ii) La crianza de cuyes: interesante por su conjugación con la actividad agrícola como fuente de materia orgánica para el suelo (FelipeMorales, 2002), la alimentación (carne) y la provisión de energía doméstica (biogas). Todos los subproductos de esta actividad se usan en la misma finca, con su carne satisfacen necesidades domésticas de la familia, incluso genera excedentes para la venta; (iii) Elaboración de compost: a partir de todos los desechos orgánicos tanto de la actividad agrícola y doméstica aprovechados para la producción de compost que es utilizado en el abonamiento del suelo de la finca, y genera excedentes que son vendidos, generando ingresos adicionales. En esta finca "la basura no existe" indican los dueños; (iv) biodigestor: tienen implementado un Biodigestor modelo chino con capacidad de $6 \mathrm{~m} 3$, el cual según lo indican los dueños produce en promedio $3 \mathrm{~m} 3$ de gas/día; éste es alimentado periódicamente 
y descargado una vez al año, se obtienen como subproductos biol, abono orgánico y biogás; (v) humedal artificial: tecnología limpia usada para la descontaminación y reutilización de aguas servidas generadas por el consumo doméstico en la finca, constituyendo una alternativa necesaria ante la necesidad de agua y evitando la contaminación ambiental.

De la información rescatada de esta experiencia compartida por la finca BioAgricultura, donde se evidencia el enfoque sistémico, la diversificación productiva no sólo a nivel de producción agrícola sino también de servicios, otorga un valor tanto ecológico, social, y principalmente demuestra económico (M. A. Altieri \& Yurjevic, 1992), configurando así una posibilidad factible de hacer agricultura sostenible.

Ferias agroecológicas en la ciudad de Lima

De la revisión en noticias, literatura gris y apuntes tomados en un curso de Agroecología Avanzada en la Universidad Nacional Agraria la Molina (FelipeMorales, 2020; NETLima, 2019), las bioferias o ferias ecológicas se han implementado desde hace varios años en
Lima y otras ciudades del país, tal es el caso de la Bioferia de Miraflores, en La Molina, en Surquillo, en San Isidro, en Barranco, entre otras, que han iniciado como iniciativas de grupos ecológicos. Estas bioferias vienen siendo apoyadas por las municipales distritales, así como en parte por la Municipalidad Metropolitana de Lima. Uno de estos actores de interés que ha movilizado los temas agroecológicos y la organización de pequeños agricultores ecológicos en nuestro país es la Asociación Nacional de Productores Ecológicos del Perú-ANPE PERÚ, que a la actualidad cuenta con 20 bases regionales y 172 organizaciones locales (ANPE-PERU, n.d.).

Como ya se indicó en párrafos antes, el actor de interés más importante que se ha sumado en los últimos años a la promoción de las bioferias ha sido APEGA (Fernando Alvarado; Siura Saray, 2015), que ha promovido las Ferias Agropecuarias Mistura-FAM, una de las más importantes de éstas es la Feria que se ha venido desarrollando en la Av. Brasil, Magdalena del Mar; sin embargo, a la actualidad esta Feria ha sido dejada por APEGA para que se administrada por un comité de gestión independiente. Aunque esta Feria no es netamente orgánica o ecológica, si busca ofertar productos agropecuarios frescos y 
una venta directa para pequeños productores agropecuarios.

$\mathrm{Al}$ año 2020, en un escenario de pandemia por el COVID 2019, las ferias no están funcionando como venían haciéndolo, sin embargo, algunas de ellas ha venido adecuándose a una atención por delivery; todo esto indica que las ferias agroecológicas o bioferias y los mismos agricultores tienen que adecuarse a un nuevo escenario que podría ser incluso más potencial que el escenario de las ferias físicas, pasando a la venta online, usando redes sociales y diversos modelos de comercio electrónico que a la actualidad están muy desarrollados (Robles, 2015). Una experiencia vigente y actual, puesta en marcha desde España, que se ha difundido en varios países de la Unión Europea y con expansión a Latinoamérica es la empresa llamada "La Colmena que Dice Sí", ésta facilita la puesta en mercado de productores ecológicos a través de una plataforma virtual y de e-commerce, ello puede ser un ejemplo a ser imitado por las bioferias y pequeños productores peruanos (La-Colmena-que-Dice-Si, n.d.).

Entre los aspectos más importantes y destacables que puedo analizar de este esquema de comercialización por las Bioferias, puedo citar los siguientes: (i) Acercamiento directo entre el productor agropecuario y el consumidor: Esto fortalece los lazos sociales y de solidaridad de los pobladores de las urbes los con pobladores de zonas vulnerables de pobreza, de donde en general vienen para ofrecer sus productos en estas ferias; (ii) oferta de productos frescos para la alimentación: Debido a que se obvia parte de la cadena de comercialización (comerciantes intermediarios), la llegada a los consumidores de los productos desde las parcelas de producción es más rápida; (iii) permiten una alimentación saludable y con responsabilidad ambiental: Esto debido a que los productos expendidos en estas bioferias son en su mayoría ecológicos u orgánicos, los cuales tienen un proceso productivo y post productivo responsable con el ambiente y con limitado o nulo uso de pesticidas o agrotóxicos.

\section{RESULTADOS}

De la revisión realizada se puede concluir que la agroecología constituye en una alternativa factible para ser promovida e implementada en un sistema agrario dominado por la pequeña agricultura, debiendo promoverse por los gobiernos nacionales, regionales, locales y por las mismas organizaciones de productores. 
Las bioferias o ferias ecológicas son modelos que facilitan el desarrollo de la agroecología, trayendo beneficios win to win tanto para el pequeño productor como para el consumidor en las ciudades, sin embargo, en un contexto de pandemia y post COVID19 deberá adecuarse hacia nuevas alternativas de comercio electrónico en ambientes virtuales.

\section{REFERENCIAS BIBLIOGRÁFICAS}

Akaev, A. A., \& Sadovnichii, V. A. (2010). Mathematical model of population dynamics with the world population size stabilizing about a stationary level. Doklady Mathematics, 82(3), 978-981.

https://doi.org/10.1134/S1064562410 060360

Alegre, J. (2017). La agroforestería en la amazonía peruana para recuperar suelos degradados y mitigar efectos del cambio climático. Presentado En «Crianza Del Suelo Para El Buen Vivir» XVI Congreso Nacional y VII Ingternacional de La Ciencia Del Suelo.

Altieri, M. A., \& Yurjevic, A. (1992). La agroecología y el desarrollo rural sostenible en América Latina. https://repositorio.cepal.org/handle/1 $1362 / 33761$

Altieri, M.A. (1987). Agroecology: the scientific basic of alternative agriculture. WestviewPress. https://www.cabdirect.org/cabdirect/ abstract/19896768581

Altieri, Miguel A., \& Nicholls, C. I. (2020). Agroecology and the emergence of a post COVID-19 agriculture. Agriculture and Human Values, $37(3)$, $525-526$. https://doi.org/10.1007/s10460-02010043-7

ANPE-PERU. (n.d.). Sobre ANPE PERU. Web. Retrieved September 17, 2020, from https://www.anpeperu.org/

Barham, B. L., \& Weber, J. G. (2012). The Economic Sustainability of Certified Coffee: Recent Evidence from Mexico and Peru. World Development, 40(6), 1269-1279. https://doi.org/10.1016/j.worlddev.20 11.11.005

Beuchelt, T. D., \& Zeller, M. (2011). Profits and poverty: Certification's troubled link for Nicaragua's organic and fairtrade coffee producers. Ecological Economics, 70(7), 1316-1324. 
https://doi.org/10.1016/j.ecolecon.20 11.01 .005

Callicott, J. B. (1988). Agroecología en contexto. Journal of Agricultural Ethics, $\quad 1, \quad 3-9$. https://doi.org/https://doi.org/10.100 7/BF02014458

Cuesta, E. O., Buley, M., Serrano, M., \& Montenegro, L. (1998). Comercialización de la producción orgánica en América Latina. Agricultura Orgánica, 4.

Davies, F. T., Calderón, C. M., \& Huaman, Z. (2005). Influence of arbuscular mycorrhizae indigenous to Peru and a flavonoid on growth, yield, and leaf elemental concentration of "Yungay" potatoes. HortScience, 40(2), 381385.

https://doi.org/10.21273/hortsci.40.2. 381

Dilas-Jiménez, J. O., \& Mugruza-Vassallo, C. (2020). Instalación De Fincas Cafetaleras En Sistema Agroforestal Para Recuperación Y Sostenibilidad De Suelos Degradados De Selva Alta. Revista de Investigación En Agroproducción Sustentable, 4(1). https://doi.org/http://dx.doi.org/10.25 127/aps.20201.534
Epule, T. E., \& Bryant, C. R. (2017). The adoption of agroecology and conventional farming techniques varies with socio-demographic characteristics of small-scale farmers in the fako and meme divisions of cameroon. GeoJournal, 82(6), 11451164.

https://doi.org/10.1007/s10708-0169734-y

Estado-Peruano. (2008). Ley $N^{\circ}$ 29196, Ley de Promoción de la Producción Orgánica $\quad y \quad$ Ecológica. https://cdn.www.gob.pe/uploads/doc ument/file/385599/Ley_N_2919620 191013-25586-1uyy2tc.pdf

Estado-Peruano. (2012). Decreto Supremo $N^{\circ} \quad$ 010-2012-AG, Aprueban reglamento de la Ley $N^{\circ}$ 29196, Ley de Promoción de la Producción Orgánica $\quad y \quad$ Ecológica. https://cdn.www.gob.pe/uploads/doc ument/file/385599/Ley_N_2919620 191013-25586-1uyy2tc.pdf

Estado-Peruano. (2019). Ley $N^{\circ} 30983$, Ley que modifica la Ley $N^{\circ} 29196$, ley de promoción de la producción orgánica o ecológica, a fin de desarrollar la certificación de productos orgánicos producidos por pequeños productores. 
https://busquedas.elperuano.pe/norm aslegales/ley-que-modifica-la-ley29196-ley-de-promocion-de-laproduc-ley-n-30983-1789945-2/

Felipe-Morales, C. F. (n.d.). Hacia una agricultura sustentable en el Perú.

Felipe-Morales, C. F. (2002). Manejo Agroecológico del Suelo en Sistemas Andinos. Agroecologia: El Camino Hacia Una Agricultura Sustentable, Ediciones Científicas Americana, 15.

Felipe-Morales, C. F. (2020). Agroecología Avanzada.

Fernando Alvarado; Siura Saray. (2015). Peru: historia del movimiento agroecologico 1980-2015 1. 10(2), 77-84.

García, A., Laurín, M., Llosá, M., Gonzálvez Pérez, V., Sanz, M., \& Porcuna y Col, J. (2006). Contribución de la agricultura ecológica a la mitigación del cambio climático en comparación con la agricultura convencional. Agroecología, 1, 75-87.

González Elizondo, M., Jurado Ybarra, E., González Elizondo, S., Aguirre Calderón, Ó., Jiménez Pérez, J., \& Návar Cháidez, J. (2003). Cambio climático mundial: origen $y$ consecuencias.

IFOAM. (2008). Definition of Organic Agriculture. IFOAM-Organics International. https://www.ifoam.bio/whyorganic/organiclandmarks/definition-organic

La-Colmena-que-Dice-Si. (n.d.). $\quad L a$ Colmena que Dice Sí. Retrieved September 17, 2020, from https://lacolmenaquedicesi.es/es

Laura S., A. B. (2013). Evaluación de la sustentabilidad de la producción orgánica el café a través de la medición de eficiencia económica con variables ambientales. Natura@economía, $\quad$ 1(2), 91. https://doi.org/10.21704/ne.v1i2.142 4

Migliorini, P., \& Wezel, A. (2017). Converging and diverging principles and practices of organic agriculture regulations and agroecology. A review. Agronomy for Sustainable Development, 37(6). https://doi.org/10.1007/s13593-0170472-4 
MINAM. (2015). Casa Blanca: El pequeño paraíso de Carmen y Ulises. Ministerio del Ambiente. youtube.com/watch? $\mathrm{v}=\mathrm{pWeDYqFse}$ bQ

NETLima. (2019). Bioferias en Lima. Web. https://www.netlima.com/lugardeta.php?pcamp $0=5243$

Nuñez, M., Santillana, N., \& Zuñiga, D. (2005). Evaluación de cuatro cepas de Rhizobium en Vicia faba L. var. rojo mantaro en condiciones de campo. Naturaleza y Desarrollo, 3(2), 41-47.

Pabón, J. D., \& Nicholls, R. S. (2005). El cambio climático y la salud humana. 25(1), 5.8 . https://www.redalyc.org/pdf/843/843 25101.pdf

PopulationCity. (n.d.). Mundo población 1800-2100. Population.City. Retrieved September 17, 2020, from http://poblacion.population.city/worl d/

Poudel, K. L., Johnson, T. G., Yamamoto, N., Gautam, S., \& Mishra, B. (2015). Comparing technical efficiency of organic and conventional coffee farms in rural hill region of Nepal using data envelopment analysis
(DEA) approach. Organic Agriculture, 5(4), 263-275. https://doi.org/10.1007/s13165-0150102-X

Robles, J. (2015). Local markets and digital technologies: Among e-commerce and new forms of conviviality. Anthropologica, 32(33), 137-161.

Rojo-Martínez, G. E., Jasso-Mata, J., \& Velásquez-Martinez, A. (2003). Las masas forestales como sumideros de CO2 ante un cambio climático global. Revista Chapingo. Serie Ciencias Forestales y Del Ambiente, 9(1), 5767.

https://www.redalyc.org/pdf/629/629 90106.pdf

Santillana, N., Arellano, C., \& Zúñiga, D. (2016). CAPACIDAD DEL Rhizobium DE PROMOVER EL CRECIMIENTO EN PLANTAS DE TOMATE (Lycopersicon esculentum Miller. Ecología Aplicada, 4(1-2), 47. https://doi.org/10.21704/rea.v4i12.297

Sierra-Productiva. (n.d.). Programa Sierra Productiva. Portal Web. Retrieved September 17, 2020, from https://www.sierraproductiva.org/ 
Sierra-Productiva. (2016). Sierra

Productiva-reportaje. $\quad 300$ Sesenta

Comunicaciones.

https://www.youtube.com/watch?v=

bUB9tnP54CM\&ab_channel=300Se

sentaComunicaciones

Wezel, A., Bellon, S., Doré, T., Francis, C., Vallod, D., \& David, C. (2009). Agroecology as a science, a movement and a practice. Sustainable Agriculture, 2, 27-43. https://doi.org/10.1007/978-94-0070394-0_3

\section{Contacto:}

Mg. Josue Otoniel Dilas-Jiménez

jdilas@unat.edu.pe 